\title{
Quantum spatial search on graphs subject to dynamical noise
}

\author{
Marco Cattaneo, ${ }^{1}$ Matteo A. C. Rossi, ${ }^{2}$ Matteo G. A. Paris, ${ }^{1}$ and Sabrina Maniscalco ${ }^{2,3}$ \\ ${ }^{1}$ Quantum Technology Lab, Dipartimento di Fisica "Aldo Pontremoli”, Università degli Studi di Milano, I-20133 Milano, Italy \\ ${ }^{2}$ QTF Centre of Excellence, Turku Centre for Quantum Physics, Department of Physics and Astronomy, University of Turku, \\ FI-20014 Turun Yliopisto, Finland \\ ${ }^{3}$ QTF Centre of Excellence, Department of Applied Physics, School of Science, Aalto University, FI-00076 Aalto, Finland
}

(Received 7 September 2018; published 27 November 2018)

\begin{abstract}
We address quantum spatial search on graphs and its implementation by continuous-time quantum walks in the presence of dynamical noise. In particular, we focus on search on the complete graph and on the star graph of order $N$, also proving that noiseless spatial search shows optimal quantum speedup in the latter, in the computational limit $N \gg 1$. The noise is modeled by independent sources of random telegraph noise (RTN), dynamically perturbing the links of the graph. We observe two different behaviors depending on the switching rate of RTN: fast noise only slightly degrades performance, whereas slow noise is more detrimental and, in general, lowers the success probability. In particular, we still find a quadratic speedup for the average running time of the algorithm, while for the star graph with external target node, we observe a transition to classical scaling. We also address how the effects of noise depend on the order of the graphs and discuss the role of the graph topology. Overall, our results suggest that realizations of quantum spatial search are possible with current technology and indicate the star graph as the perfect candidate for the implementation by noisy quantum walks, owing to its simple topology and nearly optimal performance for just a few nodes also.
\end{abstract}

DOI: 10.1103/PhysRevA.98.052347

\section{INTRODUCTION}

Quantum spatial search [1] is the problem of finding a marked element in a structured database, i.e., a database whose items are connected by a structure of links mimicking a graph. Essentially, it is the generalization of the Grover algorithm [2] to search problems in which one has to take into account the spatial organization of the dataset.

Childs and Goldstone showed that an algorithm based on continuous-time quantum walks (CTQWs) [3] may solve the problem of quantum spatial search on certain graph topologies in a time $T=O(\sqrt{N})$ [4], where $N$ is the order of the graph, thus outperforming any classical algorithm, where the searching time is bounded to $T=O(N)$. In particular, they proved that the full speedup of the order of $T=O(\sqrt{N})$ is achieved in the case of the complete graph, the hypercube graph, and the $d$-dimensional lattice for $d \geqslant 4$.

In recent years, many other graph topologies have been considered. For instance, the algorithm has been investigated on complete bipartite graphs [5], balanced trees [6], ErdösRényi graphs [7,8], the simplex of the complete graph [9], and graphs with fractal dimensions [10,11]. Moreover, it has been shown that high connectivity and global symmetry of the graph are not necessary for fast quantum search [12,13]. The first result of this paper is a proof of the optimality of quantum spatial search on the star graph, both when the target is the central node and when it is one of the external ones.

These results are very promising, but in order to address concrete implementations, one should consider the presence of noise and disorder in the system. In particular, one should analyze the effect of noise on the success probability of the algorithm and on the scaling of the searching time. As a matter of fact, the study of the effects of noise on spatial search is still at the early stages. The robustness against noise upon considering adiabatic quantum computation has been studied [14], as well as the performance of spatial search on graphs with broken links [5]. More recently, it has been shown that the coupling to a thermal bath may improve the efficiency of the algorithm in the presence of static disorder [15], whereas a fully dynamical description of the noise is still missing. The search algorithm has been analyzed on random temporal networks [16], i.e., Erdös-Rényi graphs whose topology changes after a certain time interval, though this model can hardly mimic the dynamics of real noise.

In this paper, we address continuous-time quantum spatial search on graphs subject to dynamical noise. In particular, we analyze the performance of the algorithm on the complete graph and on the star graph, after having analytically proven that the search is also optimal on the latter. The noise is modeled as random telegraph noise (RTN) affecting the links of the graph with tunable strength, ranging from a weak perturbation of the hopping amplitudes to a strength comparable to the coupling, inducing dynamical percolation. Our choice for the noise is motivated by its relevance in systems of interest for quantum information processing [17-21] and by the fact that RTN is at the root of the $1 / f$ noise affecting superconducting qubits [22]. In recent years, some works have addressed the properties of CTQWs on the one-dimensional lattice subject to random telegraph noise [23-26], also in the presence of spatial correlations [27]. In this paper, we analyze the effects of RTN on spatial search on graphs with generic topology. Other models of a CTQW subject to dynamical noise have been proposed as well [28-30]. 
The paper is structured as follows: in Sec. II, we review the continuous-time quantum spatial search algorithm and we prove its optimality on the star graph. In Sec. III, we introduce the noise model and we discuss the noisy evolution of the walker. In Sec. IV A, we present our results on the effects of noise on the complete graph, while in Sec. IV B, we focus on the star graph. Section V closes the paper with some concluding remarks.

\section{THE ALGORITHM}

Given a certain graph $G$ composed of $N$ nodes, we want to find the marked element $w$, called the target node. The graph $G$ is described by the adjacency matrix $A$, whose elements are defined as

$$
A_{i j}= \begin{cases}1 & \text { if nodes } i, j \text { connected } \\ 0 & \text { otherwise. }\end{cases}
$$

The Hilbert space of the walker is $\mathcal{H}=\operatorname{span}\{|j\rangle\}$ with $j=$ $1, \ldots, N$, where $|j\rangle$ is the single-particle localized state associated to the node $j$. The Hamiltonian of the algorithm reads

$$
H=\gamma L+H_{w}=\gamma L-|w\rangle\langle w|
$$

where $H_{w}=-|w\rangle\langle w|$ is called the oracle Hamiltonian, $\gamma$ is a suitable coupling constant, and we introduced the Laplacian matrix $L=D-A$, where $D$ is the degree matrix, a diagonal matrix where the $i$ th entry is the degree of the $i$ th node, i.e., the number of links connected to it. Notice that we are neglecting an overall constant in $H$, which fixes the unit of measure for time $t$ and related quantities.

The quantum walk starts in the fully delocalized state $|s\rangle$, where

$$
|s\rangle=\frac{1}{\sqrt{N}} \sum_{j=1}^{N}|j\rangle,
$$

and the state at time $t$ reads

$$
|\psi(t)\rangle=e^{-i H t}|s\rangle .
$$

After the time $t$, we measure in the vertices basis. The probability of measuring the walker in the target node is $p_{w}(t)=|\langle w \mid \psi(t)\rangle|^{2}$. We define the success probability $p_{\text {succ }}$ as the maximum probability,

$$
p_{\text {succ }}=\max _{t} p_{w}(t),
$$

and $T$ the smallest time instant for which $p_{\text {succ }}$ is achieved. Optimizing the search algorithm then consists in finding $\gamma$ such that for $T$ as small as possible, the success probability $p_{\text {succ }}=$ is maximal. We say that the algorithm is optimal if $p_{\text {succ }} \approx 1$ in a time $T=O(\sqrt{N})$.

As an example, we review the performance of the algorithm on the complete graph, which had already been addressed employing a different computational framework as the "analog analogue" of Grover's algorithm [31]. The action of the Hamiltonian on the states $|s\rangle$ and $|w\rangle$, using as $L$ in Eq. (2) the Laplacian of the complete graph and choosing $\gamma=1 / N$, reads

$$
H|s\rangle=-\frac{1}{\sqrt{N}}|w\rangle, \quad H|w\rangle=-\frac{1}{\sqrt{N}}|s\rangle .
$$

Therefore, the Hamiltonian drives transitions between the two states and after a time $T=\pi \sqrt{N} / 2$, we have $|\psi(T)\rangle=|w\rangle$, i.e., the algorithm is optimal for any order $N$.

\section{Optimality of the star graph}

Let us now address the proof of the optimality of the algorithm on the star graph. The search on the star graph has already been investigated in [5] as a particular case of complete bipartite graphs, and a success probability $p_{\text {succ }} \approx$ $1 / 2$ in a time $T=O(\sqrt{N})$ was found. However, in [5], the authors choose to use the adjacency matrix instead of the Laplacian operator in the Hamiltonian of the algorithm given by Eq. (2). This choice is irrelevant if the graph is regular, as the elements on the diagonal of $L$ are all equal and are thus just a energy shift, but it leads to completely different dynamics in nonregular graphs [32], as is the case with the star graph.

In what follows, we prove that the continuous-time quantum spatial search is optimal on the star graph, if we employ the Laplacian as in Eq. (2). The star graph consists of $N-1$ nodes connected to a central node. There are two different situations to consider: the case in which the target node $w$ is the central one and the case in which the target node is one of the external nodes.

Let us start with the case in which the target node is the central node of the star graph, named $|c\rangle$. If $|c\rangle=|w\rangle$, by choosing $\gamma=1 / N$, we obtain

$$
H|s\rangle=-\frac{1}{\sqrt{N}}|w\rangle, \quad H|w\rangle=-\frac{1}{\sqrt{N}}|s\rangle,
$$

and therefore the dynamics is analogous to the one on the complete graph and we find the target node with $p_{\text {succ }}=1$ after $T=\pi \sqrt{N} / 2$, independently of the order $N$ of the graph.

The proof of the optimality when the target is one of the external nodes is more involved and is extensively addressed in the Appendix. By making use of the Krylov subspace method to reduce the space of the walker [5] and then by employing degenerate perturbation theory [12], we show that in the computational limit $N \gg 1,|\psi(T)\rangle=|w\rangle+O\left(N^{-1 / 2}\right)$ at time $T=\pi \sqrt{N} / 2$, i.e., the algorithm is optimal. Notice that in this case, we have to choose $\gamma=1$ for the algorithm to succeed.

While the proof shows the optimality of the algorithm for large $N$, Fig. 1 shows that the success probability is also close to 1 for small values of $N, p_{\text {succ }} \approx 1-N^{-2}$, and the optimal time scales as $\sqrt{N}$. This suggests that the star graph may be a good candidate for an experimental implementation of continuous-time quantum spatial search since just a few nodes are required to achieve quantum speedup. Furthermore, the star graph has a simpler topology compared to the other graphs that are suitable for spatial search, and thus it might be easily realized in a laboratory using, e.g., superconducting circuits. 


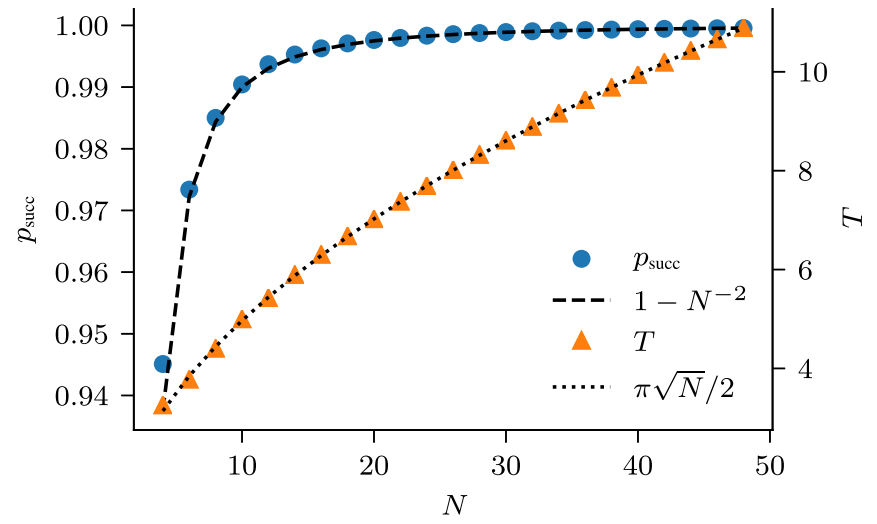

FIG. 1. Success probability $p_{\text {succ }}$ (blue circles, left axis) and optimal time $T$ (orange triangles, right axis) of quantum spatial search on the star graph as a function of $N$ with an external node as the target. The dots represent the exact quantities, the dotted line is the benchmark $\pi \sqrt{N} / 2$, and the dashed line represents the function that better approximates $p_{\text {succ }}$, which we have found to be $1-N^{-2}$, showing that the remainders in Eq. (A11) are actually of the order of $N^{-1}$ rather than $N^{-1 / 2}$. The plot shows that even for finite $N$, the success probability is very high and that the optimal time follows the asymptotic behavior.

\section{THE NOISE MODEL}

The random telegraph noise (RTN) is the continuous-time stochastic process that describes the dynamics of a bistable fluctuator, i.e., a quantity which switches randomly between two given values (say \pm 1 ) according to a certain switching rate $\mu$. The RTN is completely characterized as $\{g(t), t \in$ $[0,+\infty)\}$, where $g(t)= \pm 1$, which implies that the probability of switching $n$ times in a time $t$ follows a Poisson distribution,

$$
p_{\mu}(n, t)=e^{-\mu t} \frac{(\mu t)^{n}}{n !} .
$$

The stochastic process is stationary and its autocorrelation function reads

$$
\langle g(\tau) g(0)\rangle=e^{-2 \mu|\tau|},
$$

corresponding to a Lorentzian spectrum.

Motivated by the kind of noise observed in superconducting networks, we model the environmental noise by assuming that the links of the graph are affected by independent and equal (i.e., with the same switching rate $\mu$ ) RTN. Accordingly, we modify the Laplacian operator in Eq. (2), keeping the classical probability conservation rule for which the sum of the elements in a column of the Laplacian matrix is zero.

The noise is described by the $N \times N$ matrix $\mathbf{g}(t)$, where $N$ is the number of nodes in the graph and $g_{j k}(t)$ is the stochastic process describing the noise on the link connecting $j$ to $k$. The matrix $\mathbf{g}(t)$ is thus symmetric, zero diagonal, and has only $l$ independent entries, where $l$ is the number of links in the graph. Since the noises on different links are independent of each other, we have for the non-zero entries of $\mathbf{g}(t)$

$$
\left\langle g_{j k}(\tau) g_{j^{\prime} k^{\prime}}(0)\right\rangle=e^{-2 \mu|\tau|}\left(\delta_{j j^{\prime}} \delta_{k k^{\prime}}+\delta_{j k^{\prime}} \delta_{k j^{\prime}}\right) .
$$

We now replace Eq. (2) with a noisy Hamiltonian depending on the stochastic process $\mathbf{g}(t)$. The noisy Laplacian $L^{(\mathbf{g})}(t)$ in the node basis reads

$$
L_{j k}^{(\mathbf{g})}(t)= \begin{cases}-\left[1+v g_{j k}(t)\right] & \text { if }(j, k) \text { connected } \\ D_{j k}+v \sum_{i=1}^{N} g_{i k}(t) & \text { if } j=k \\ 0 & \text { otherwise }\end{cases}
$$

where $v$ is the noise strength and $D$ is the degree matrix. If $v=1$, we obtain dynamical percolation, i.e., the random creation and removal of links in the graph according to the switching rate $\mu$. The Hamiltonian then reads

$$
H^{(\mathbf{g})}(t)=\gamma L^{(\mathbf{g})}(t)-|w\rangle\langle w| .
$$

If the initial state of the walker is $\rho_{0}=|s\rangle\langle s|$, the evolved density matrix is the ensemble average,

$$
\rho(t)=\left\langle U(t) \rho_{0} U(t)^{\dagger}\right\rangle_{\{\mathbf{g}(t)\}},
$$

where $\langle\cdot\rangle_{\{\mathbf{g}(t)\}}$ denotes the average over all possible realizations of the stochastic process $\mathbf{g}(t)$ and $U(t)$ is the unitary evolution operator associated to a particular realization, given by

$$
U(t)=\mathcal{T} \exp -i \int_{0}^{t} d s H^{(\mathbf{g})}(s),
$$

where $\mathcal{T}$ is the time-ordering operator.

Equation (13) defines a map that describes the dynamics of the open quantum system, and $\rho(t)$ is the only relevant physical quantity for investigating the evolution of the system. From this point of view, the noise model discussed above is just an effective microscopic description of the coupling between system and environment that generates the quantum map that we are actually observing.

The success probability at time $T$ is now the matrix element

$$
p_{\text {succ }}=\langle w|\rho(T)| w\rangle \text {. }
$$

\section{QUANTUM SPATIAL SEARCH ON NOISY GRAPHS}

In this section, we discuss how random telegraph noise affects continuous-time quantum spatial search on the complete and the star graphs.

\section{A. Complete graph}

The time evolution of the walk, given by Eq. (13), cannot be computed analytically for a large number of links; therefore, we simulate the dynamics numerically and then we average over a big number of realizations of the noise. Since this is the optimal value in the noiseless case, we set $\gamma=1 / N$ in Eq. (11). The code used in this work is written in JULIA [33] and is available on GitHub [34]. Since the number of noise trajectories explored in the simulation is finite, fluctuations are present on the mean value leading to the quantum map given by Eq. (13). We have calculated the standard deviation of this mean value and considered a number of noise realizations that is large enough to make such standard deviation irrelevant, i.e., nonvisible in the graphs. In particular, after this careful analysis of numerical uncertainties, we have chosen to average over 10000 realizations of the noise when $\mu \geqslant 1$, and over 20000 when $\mu<1$. 


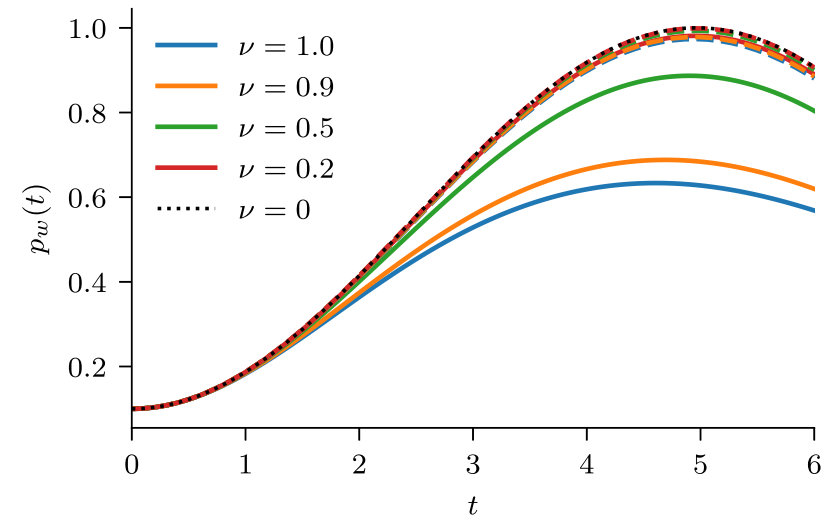

FIG. 2. Probability of measuring the target node $p_{w}(t)=$ $\langle w|\rho(t)| w\rangle$ as a function of time $t$ on the complete graph of or$\operatorname{der} N=10$, for fast noise $(\mu=10$, dashed lines) and slow noise ( $\mu=0.01$, solid lines). Different values of the noise strength, $v=$ $0.2,0.5,0.9$, and 1.0, are shown, respectively, from top to bottom, by the red, green, orange, and blue lines. The dotted black line describes the noiseless case $(v=0)$. Fast noise barely affects the algorithm, while slow noise decreases the success probability, but also the optimal time.

In order to analyze the robustness of the search in the presence of noise, we focus on the success probability of the algorithm and on the optimal time $T$. We explore several scenarios by varying three fundamental parameters: the order of the graph $N$, the noise strength $\nu$, and the switching rate $\mu$ of the RTN. In particular, we identify two different regions of values of the switching rate, and we call the RTN with $\mu \lesssim 1$ slow or semistatic noise, and we call the RTN with $\mu \gtrsim 1$ fast noise. In Fig. 2, we plot the probability of measuring the target node $p_{w}(t)=\langle w|\rho(t)| w\rangle$ as a function of time, for $N=10$ and choosing $\mu=10$ and $\mu=0.01$. Several values of the noise strength $v$ are considered.

A clear difference in the behaviors appears, depending on the value of the switching rate $\mu$ : for fast noise, the algorithm is still optimal, in the sense that we obtain a success probability (i.e., the maximal probability of measuring the target node) $p_{\text {succ }} \approx 1$ in a time $T \approx \pi \sqrt{N} / 2$; on the contrary, slow noise significantly affects the efficiency of the search, and for $\mu=0.01$ and $\nu=1$ the probability of success is around $60 \%$. At any time, the probability of measuring the target node for a fixed switching rate and a certain noise strength $v$ is always lower than for a smaller noise strength, proving that in general the presence of dynamical noise jeopardizes the algorithm. The left panel of Fig. 3 depicts the success probability as a function of the switching rate $\mu$, showing that decreasing the switching rate of the noise leads to worse and worse performance.

For higher orders of the graph, we have obtained qualitatively similar results, although increasing the order leads to slightly better success probabilities. This is intuitive since by adding nodes to the complete graph we are creating more possible paths connecting each node to the target, decreasing the effects of broken links due to semistatic noise. The success probability as a function of $N$ is depicted in the central panel of Fig. 3 for slow noise and several values of $v$. Further analysis suggests that changing the value of the coupling constant $\gamma$ in the presence of noise does not improve the results of the spatial search, but the optimal value remains $\gamma=1 / N$ as in the noiseless case.

It should be noticed that while the success probability tragically decreases in the presence of semistatic noise, the time $t_{\max }$ at which we find the maximal success probability is slightly smaller. From a computational point of view, one may assume the possibility of "recognizing" the outcome of the spatial search algorithm, being able to tell whether or not it is the right solution. In this framework, we are allowed to run more trials of the algorithm until the correct solution is found. The probability of getting the right target node at the $n$th trial is given by

$$
p_{g}(n)=\left(1-p_{\text {succ }}\right)^{n-1} p_{\text {succ }} .
$$

Equation (16) is a geometric distribution with mean value $\langle n\rangle=1 / p_{\text {succ }}$. Therefore, the average optimal time $T$ of the algorithm with success probability $p_{\text {succ }}$ is given by

$$
T=\frac{t_{\text {max }}}{p_{\text {succ }}} .
$$
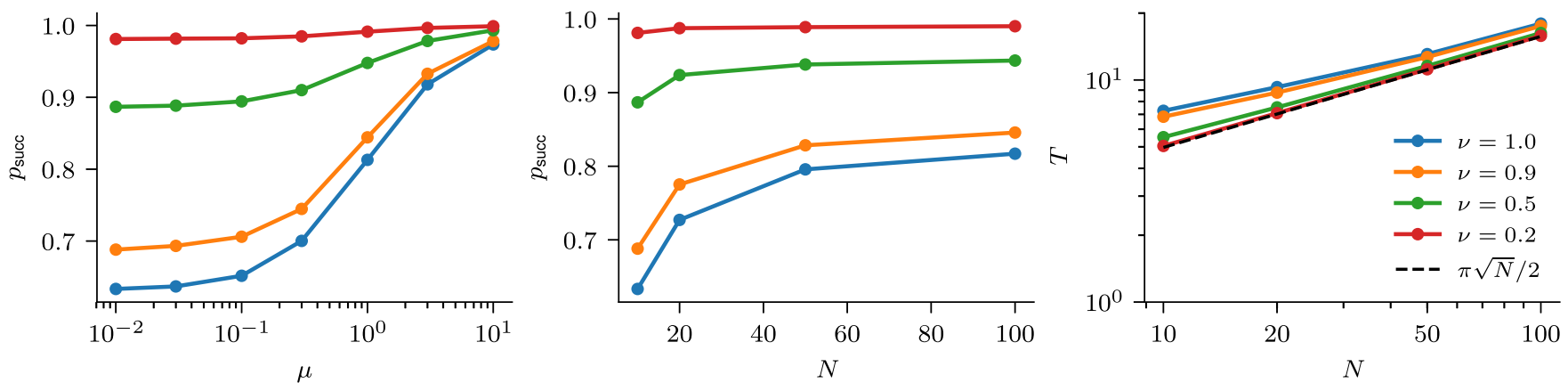

FIG. 3. The left panel shows the success probability $p_{\text {succ }}$ of the quantum spatial search on the complete graph of order $N=10$, as a function of the switching rate $\mu$. The central panel and right panel show, respectively, the success probability and the average running time $T$ on the complete graph as a function of $N$ for slow noise $(\mu=0.01)$. In the three plots, different values of the noise strength, $v=0.2,0.5,0.9$, and 1 , are shown, respectively (from top to bottom in the first two panels, from bottom to top in the last one), by the red, green, orange, and blue lines. The success probability decreases with the noise strength, but it increases with $N$. Although the success probability is lower than 1 , the average running time is proportional to $\sqrt{N}$ (showed with a black dashed line), and thus the algorithm can still outperform a classical one for sufficiently large $N$. 

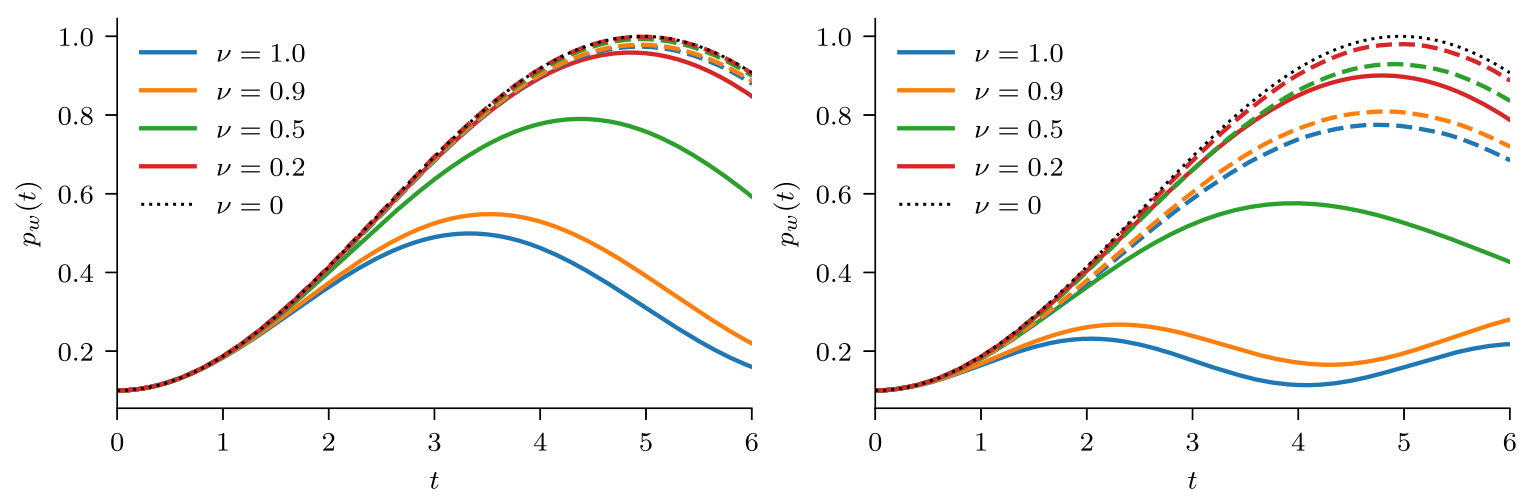

FIG. 4. Probability of measuring the target node $p_{w}(t)=\langle w|\rho(t)| w\rangle$ as a function of time, on the star graph of order $N=10$ with central node (left) and an external node (right) as the target, for slow noise (solid lines, $\mu=0.01$ ) and fast noise (dashed lines, $\mu=10$ ). Different values of the noise strength, $v=0.2,0.5,0.9$, and 1 , are represented by the red, green, orange, and blue lines (from top to bottom), respectively. The dotted black lines describe the noiseless case. If the target node is the central one, the results are qualitatively similar to the case of the complete graph, but the effect of noise is stronger. In the case of an external target node, slow noise dramatically affects the success probability.

This is the time we should compare with the optimal analog in the noiseless case. The right panel of Fig. 3 shows the results for the average optimal time $T$ as a function of $N$ for slow noise and for several values of $v$. Apart from a constant factor in the logarithmic scale, it is clear that the average optimal time still follows the quadratic speedup $T \sim O(\sqrt{N})$ for any value of noise strength. With fast noise (not shown), the curves are closer to the noiseless case.

We stress, however, that this should not lead one to underestimate the effect of noise since a success probability close to 1 is an important feature in quantum spatial search. Indeed, besides being the analog of Grover's algorithm on structured databases, quantum spatial search may have other applications and interpretations. For instance, let us consider a system composed of quantum nodes connected by links, and let us assume to know that one of the nodes is affected by a certain potential well (mimicking the oracle Hamiltonian), but without knowing where it actually is. In this case, we may find the marked node by running the quantum spatial search algorithm, but we would not be able to recognize the solution unless the success probability is close to the unit.

\section{B. Star graph}

In this section, we present the results of noisy quantum spatial search on the star graph. We first consider the case in which the target is the central node, and then the case in which the target is one of the external nodes. Indeed, the dynamics of the quantum walk in the two scenarios is remarkably different (for instance, in the former case, we set $\gamma=1 / N$, while in the latter case, we set $\gamma=1$ ) and, as we will see, the effect of the noise is different as well.

\section{Central target node}

In this case, the effect of the dynamical noise on the search algorithm is similar to the case of the complete graph. In the left panel of Fig. 4, we plot the probability of measuring the target node as a function of time for several values of $v$ and for both fast and slow noise. The optimal $\gamma$ in the presence of noise still remains $\gamma=1 / N$. Qualitatively, we obtain the same results of the previous section: fast noise lightly influences the performance of the search, while slow noise is highly detrimental, although the success probabilities are slightly lower than in the case of the complete graph.

This is easily explained, for instance, in the semistatic scenario and percolation regime: at time $t=0$, around $50 \%$ of the links of the star graph will be broken, and they will remain broken on average for almost all the evolution, since the noise is slow. Each node, apart from the central one, has only one link, and therefore it is highly probable that the walker will remain "stuck" in the isolated nodes and will not find the target node. On the contrary, in the case of the complete graph, it is very unlikely that a certain node starts with all the links cut, and therefore the walker will almost always find a path in the graph to reach the target.

This phenomenon is independent of $N$, and this is the reason why, on the star graph, increasing the order does not lead to better results for semistatic noise, as depicted in Fig. 5 (solid lines). However, the asymptotic behavior of the average

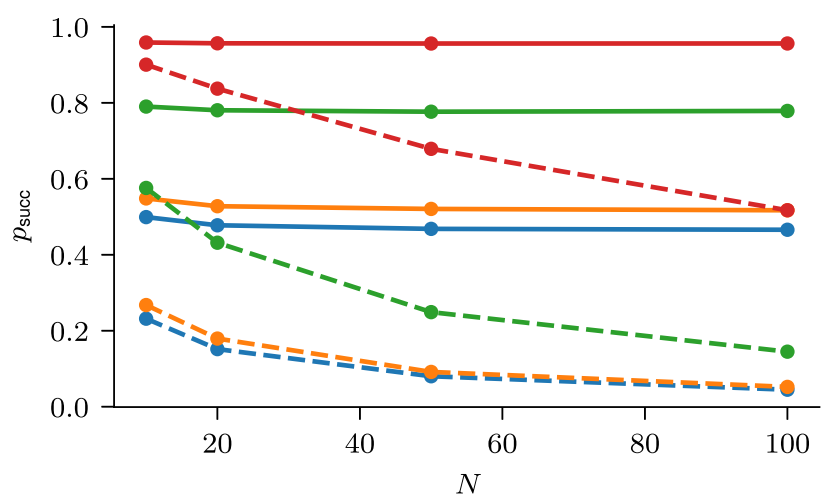

FIG. 5. Success probability on the star graph with central (solid lines) and external (dashed lines) target node, as a function of $N$, for slow noise $(\mu=0.01)$. Different values of the noise strength, $v=$ $0.2,0.5,0.9$, and 1 , are shown, respectively, from top to bottom, by the red, green, orange, and blue lines. While $p_{\text {succ }}$ remains constant with $N$ if the target node is the central one, it vanishes when the target node is external, showing how the connectivity of the target node is relevant in the presence of noise. 


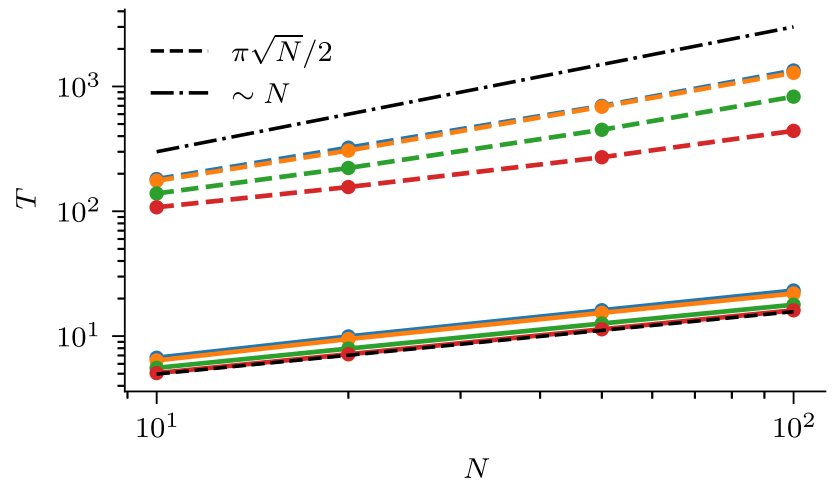

FIG. 6. Average optimal time $T$ on the star graph with central (solid lines) and external (dashed lines) target node, as a function of $N$, for slow noise with $\mu=0.01$. Different values of the noise strength, $v=0.2,0.5,0.9$, and 1 , are shown, respectively, from bottom to top, by the red, green, orange, and blue lines. The dashed black line describes the noiseless case $T=\pi \sqrt{N} / 2$, while the dot-dashed black line shows a $T \sim N$ dependence. While in the case of the central target node the algorithm still shows a quantum speedup, if the target node is external the noise makes the algorithm transition to a classical scaling with $N$.

optimal time in the framework of iterated trials, given by Eq. (17), does not change, as shown in Fig. 6 (solid lines) only for slow noise; therefore, all the considerations discussed for the complete graph still hold.

\section{External target node}

Here we analyze the effects of noise on the search on the star graph when the target node is external. Numerical analyses have suggested that decreasing the value of $\gamma$ in the presence of slow noise may lead to better performance, while when we increase the switching rate, the optimal $\gamma$ shifts toward the noiseless value 1 . However, in this work we are interested in the effects of noise on the ideal algorithm, and therefore we keep $\gamma=1$ in all the following analyses.

The results of the noisy algorithm on the star graph with external target node are shown in the right panel of Fig. 4. Qualitatively, we observe the same behavior as for the central target node, although the success probability is quantitatively much worse than in the previous cases and even fast noise affects the performance in a non-negligible way. This behavior is not surprising since the degree of the target node is only 1 ; therefore, if the noise, especially the semistatic one, affects the connecting link, then the target can no longer "exchange" probability with the rest of the graph.

Furthermore, the maximal probability of success decreases when the order of the graph increases. This might be due to the fact that as $N$ increases, the degree of the target node remains the same, while the degree of the central node, which is the only connection of the target to the rest of the graph, goes up as well, and therefore the probability current may "take the wrong direction" more easily.

Finally, we investigate how the optimal time $T$ varies in the presence of noise. Figure 6 shows that in the case of slow noise $(\mu=0.01)$, the scaling of the optimal time follows a transition from the quantum speedup $T=O(\sqrt{N})$ to the classical time
$T=O(N)$. The case of the star graph with external target node is the only one in which the noise affects both the success probability and the optimal time, once again proving that this topology is particularly weak with respect to the effects of dynamical noise.

\section{CONCLUDING REMARKS}

Quantum spatial search via continuous-time quantum walks has received much attention in the recent past. Several kinds of graphs have been proposed and their properties studied, trying to understand if and how the topology of the graph is correlated with fast spatial search. In this paper, we have taken a step further and we have proven the optimality of the algorithm on the star graph, showing that the success probability of the search is close to 1 in a optimal time $T \approx$ $\pi \sqrt{N} / 2$ also when the order $N$ of the graph is low, regardless of the position of the target node. This is particularly interesting since, owing to the simple topology of the star graph and to the feasibility of the search employing just a few nodes, our results pave the way to the experimental implementation of the continuous-time quantum spatial search algorithm.

We have also addressed the performance of quantum spatial search in the presence of dynamical noise. In particular, we have modeled the noise as a collection of bistable fluctuators with the same switching rate $\mu$, which induce independent random telegraph noise on each link of the graph. We have studied the effects of noise in several scenarios, e.g., by varying the order of the graph $N$, the switching rate $\mu$, and the noise strength $v$, and we have analyzed it on the complete graph, on the star graph with central node as the target, and on the star graph with one of the external nodes as the target. Our results show that, in general, the noise is detrimental for the probability of success of the search, while it does not affect the quadratic speedup of the time of the search $T=O(\sqrt{N})$, up to factors independent of $N$. This fact, however, should not lead one to underestimate the detrimental effect of noise since the success probability is the crucial quantity in any search algorithm.

Upon analyzing several noise scenarios, we have shown that the random telegraph noise with large switching rate, i.e., fast noise, only slightly affects the performance of the spatial search; in particular, it decreases the success probability in a nontrivial way only when applied on the star graph with one of the external nodes as the target. On the contrary, slow noise strongly jeopardizes the efficiency of the algorithm.

Finally, we have discussed how the topology of the graph plays a role in the robustness against the dynamical noise, in particular looking at the degree of the target node and at the connectivity of the graph. The complete graph, having the maximal possible connectivity and the maximal possible degree of the target node, is particularly resistant to the noise and, by increasing its order, we obtain better results since we are also increasing both the connectivity and the target degree. The star graph with central node as target is slightly more affected by the slow noise, but increasing the order does not lead to better performance since the connectivity of the graph remains the same. The star graph with one of the external nodes as target has the lowest possible connectivity and target degree, and indeed the spatial search on it is heavily 
deteriorated by the presence of dynamical noise. Increasing the order does not improve the algorithm; on the contrary, it provides worse performance since the target degree remains the same while the possible connections with the central node increase, opening more "wrong ways" for the probability current going toward the target node. While connectivity seems to be irrelevant for noiseless quantum walks [12,13], our work points out that higher connectivity of the target node plays an important role in the presence of noise.

Our analysis represents a step toward the understanding of the effects of noise in continuous-time quantum spatial search. In particular, the study of classical dynamical noise is important in view of implementing the algorithm on a physical system which is unavoidably disturbed by the external environment, as for the case of the superconducting qubits subject to $\mathrm{RTN}$ and $1 / f$ noise.

\section{ACKNOWLEDGMENTS}

M.C. was supported by the EU through the Erasmus+ programme. M.A.C.R. and S.M. acknowledge support from the Academy of Finland via the Centre of Excellence program (Project No. 312058) and Project No. 287750.

\section{APPENDIX: PROOF OF THE ASYMPTOTIC OPTIMALITY ON THE STAR GRAPH WITH EXTERNAL TARGET NODE}

Here we give the detailed proof that spatial search is optimal on the star graph when one of the external nodes is the target.

Because of the high symmetry of the star graph, it is straightforward to see that the quantum walk is confined in the Krylov subspace given by the span of the vectors $\left\{|c\rangle,|w\rangle,\left|s_{N-1}\right\rangle\right\}$, where

$$
\left|s_{N-1}\right\rangle=\frac{1}{\sqrt{N-2}} \sum_{\substack{j=1 \\ j \neq c, w}}^{N}|j\rangle .
$$

The reduced Hamiltonian in the above basis, choosing $\gamma=1$, reads

$$
H_{\mathrm{red}}=\left(\begin{array}{ccc}
N-1 & -1 & -\sqrt{N-2} \\
-1 & 0 & 0 \\
-\sqrt{N-2} & 0 & 1
\end{array}\right)
$$

We now extract a factor $N$ from the Hamiltonian in order to employ degenerate perturbation theory; we will insert it again only at the end of the proof when we will find the perturbed eigenvalues and eigenvectors. We divide the Hamiltonian into two parts, $H^{(0)}$ and $H^{(1)}$, defined as

$$
\begin{gathered}
H^{(0)}=\left(\begin{array}{ccc}
1 & 0 & -\sqrt{N-2} / N \\
0 & 0 & 0 \\
-\sqrt{N-2} / N & 0 & 0
\end{array}\right), \\
H^{(1)}=\left(\begin{array}{ccc}
-1 / N & -1 / N & 0 \\
-1 / N & 0 & 0 \\
0 & 0 & 1 / N
\end{array}\right) .
\end{gathered}
$$

The overall Hamiltonian is given by $H_{\text {red }}=H^{(0)}+H^{(1)}$, up to the factor $N$.
We must be careful in employing perturbation theory since we have to deal with two different orders, namely, $O\left(N^{-1 / 2}\right)$ and $O\left(N^{-1}\right)$, in which the second one is the square of the first one, and thus the off-diagonal elements of $H^{(0)}$ cannot be neglected in a trivial way in the series expansion of the perturbation. Therefore, we try to get to a better form of the Hamiltonian by diagonalizing $H^{(0)}$.

The eigenvalues of the Hamiltonian $H^{(0)}$ are

$$
E_{0}^{(0)}=0, \quad E_{1,2}^{(0)}=\frac{1 \mp \sqrt{1+4 / N-8 / N^{2}}}{2},
$$

with associated eigenvectors, respectively,

$$
\begin{aligned}
& \left|e_{0}\right\rangle=|w\rangle, \\
& \left|e_{1}\right\rangle=\mathcal{N}_{1}\left(-\sqrt{N} E_{1}^{(0)}|c\rangle+\left|s_{N-1}\right\rangle\right), \\
& \left|e_{2}\right\rangle=\mathcal{N}_{2}\left(-\sqrt{N} E_{2}^{(0)}|c\rangle+\left|s_{N-1}\right\rangle\right),
\end{aligned}
$$

where $\mathcal{N}_{1}$ and $\mathcal{N}_{2}$ are suitable normalization constants. Notice that for $N \gg 1\left|e_{1}\right\rangle=|s\rangle+O\left(N^{-1 / 2}\right)$, and $E_{1}^{(0)} \sim N^{-1}$, therefore $E_{0}^{(0)}$ and $E_{1}^{(0)}$ are degenerate eigenvalues in the computational limit. This is crucially important since otherwise the dynamics would remain confined near to $\left|e_{1}\right\rangle$ at any time $t$, up to factors of the order of $O\left(N^{-1 / 2}\right)$. We have chosen $\gamma=1$ exactly to get $|w\rangle$ and $\left|e_{1}\right\rangle$ asymptotically degenerate.

Being careful about the orders of the perturbation, we can now use degenerate perturbation theory [35]. First of all, we rewrite $H_{\text {red }}$ in the new basis, $\left\{|w\rangle,\left|e_{1}\right\rangle,\left|e_{2}\right\rangle\right\}$.

In the asymptotic limit $N \rightarrow \infty$, we obtain

$$
H_{\text {red }} \sim\left(\begin{array}{ccc}
0 & -N^{-3 / 2} & N^{-1} \\
-N^{-3 / 2} & N^{-2} & 2 N^{-3 / 2} \\
N^{-1} & 2 N^{-3 / 2} & 1
\end{array}\right) .
$$

We now diagonalize [up to factors of the order of $\left.O\left(N^{-2}\right)\right]$ the $2 \times 2$ matrix representing the subspace of the asymptotically degenerate eigenvectors $|w\rangle$ and $\left|e_{1}\right\rangle$. Therefore, once again, we change basis and we choose $\{(|w\rangle+$ $\left.\left.\left|e_{1}\right\rangle\right) / \sqrt{2},\left(|w\rangle-\left|e_{1}\right\rangle\right) / \sqrt{2},\left|e_{2}\right\rangle\right\}$.

In this new basis, the total Hamiltonian reads

$$
H_{\text {red }} \sim\left(\begin{array}{ccc}
-N^{-3 / 2} & -N^{-2} / 2 & N^{-1} / \sqrt{2} \\
-N^{-2} / 2 & N^{-3 / 2} & N^{-1} / \sqrt{2} \\
N^{-1} / \sqrt{2} & N^{-1} / \sqrt{2} & 1
\end{array}\right) .
$$

Eventually, we can use perturbation theory. Indeed, the offdiagonal elements can at maximum bring a contribution of the order of $O\left(N^{-2}\right)$ to the perturbed eigenvalues, while the diagonal elements are of the order of $O\left(N^{-3 / 2}\right)$. We still have off-diagonal elements in the submatrix of the asymptotically degenerate eigenvectors, but once again the contribution is of the order of $O\left(N^{-2}\right)$. Overall, this means that the ground state $\left|\lambda_{0}\right\rangle$ and the first-excited state $\left|\lambda_{1}\right\rangle$ of the Hamiltonian are

$$
\begin{gathered}
\left|\lambda_{0}\right\rangle=\left(|w\rangle+\left|e_{1}\right\rangle\right) / \sqrt{2}+O\left(N^{-1 / 2}\right), \\
\left|\lambda_{1}\right\rangle=\left(|w\rangle-\left|e_{1}\right\rangle\right) / \sqrt{2}+O\left(N^{-1 / 2}\right) .
\end{gathered}
$$

The contribution of the order of $O\left(N^{-1 / 2}\right)$ is brought by the off-diagonal elements in the submatrix of the asymptotically degenerate eigenvectors. The corresponding eigenvalues (inserting again the factor $N$ that we extracted 
at the beginning of the proof) are given by $E_{0}=-1 / \sqrt{N}+$ $O\left(N^{-1}\right), E_{1}=1 / \sqrt{N}+O\left(N^{-1}\right)$.

Therefore, in the computational limit $N \gg 1$, the evolution of the initial state reads

$$
\begin{aligned}
|\psi(t)\rangle & =e^{-i H t}|s\rangle=e^{-i H t}\left[\left|e_{1}\right\rangle+O\left(N^{-1 / 2}\right)\right] \\
& =\frac{e^{-i H t}}{\sqrt{2}}\left[\left|\lambda_{0}\right\rangle-\left|\lambda_{1}\right\rangle+O\left(N^{-1 / 2}\right)\right] \\
& =\frac{e^{-i E_{0} t}}{\sqrt{2}}\left[\left|\lambda_{0}\right\rangle-e^{-i\left(E_{1}-E_{0}\right) t}\left|\lambda_{1}\right\rangle+O\left(N^{-1 / 2}\right)\right]
\end{aligned}
$$

[1] S. Aaronson and A. Ambainis, in Proceedings of the 44th Annual IEEE Symposium on Foundations of Computer Science (IEEE, Cambridge, 2003).

[2] L. K. Grover, Phys. Rev. Lett. 79, 325 (1997).

[3] E. Farhi and S. Gutmann, Phys. Rev. A 58, 915 (1998).

[4] A. M. Childs and J. Goldstone, Phys. Rev. A 70, 022314 (2004).

[5] L. Novo, S. Chakraborty, M. Mohseni, H. Neven, and Y. Omar, Sci. Rep. 5, 13304 (2015).

[6] P. Philipp, L. Tarrataca, and S. Boettcher, Phys. Rev. A 93, 032305 (2016).

[7] S. Chakraborty, L. Novo, A. Ambainis, and Y. Omar, Phys. Rev. Lett. 116, 100501 (2016).

[8] A. Glos, A. Krawiec, R. Kukulski, and Z. Puchała, Quantum Inf. Proc. 17, 81 (2018).

[9] T. G. Wong, Quantum Inf. Proc. 15, 1411 (2016).

[10] E. Agliari, A. Blumen, and O. Mülken, Phys. Rev. A 82, 012305 (2010).

[11] S. Li and S. Boettcher, Phys. Rev. A 95, 032301 (2017).

[12] J. Janmark, D. A. Meyer, and T. G. Wong, Phys. Rev. Lett. 112, 210502 (2014).

[13] D. A. Meyer and T. G. Wong, Phys. Rev. Lett. 114, 110503 (2015).

[14] J. Roland and N. J. Cerf, Phys. Rev. A 71, 032330 (2005).

[15] L. Novo, S. Chakraborty, M. Mohseni, and Y. Omar, Phys. Rev. A 98, 022316 (2018).

[16] S. Chakraborty, L. Novo, S. Di Giorgio, and Y. Omar, Phys. Rev. Lett. 119, 220503 (2017).

[17] Y. M. Galperin, B. L. Altshuler, J. Bergli, and D. V. Shantsev, Phys. Rev. Lett. 96, 097009 (2006).

[18] B. Abel and F. Marquardt, Phys. Rev. B 78, 201302 (2008).

$$
=\frac{e^{-i E_{0} t}}{\sqrt{2}}\left[\left|\lambda_{0}\right\rangle-e^{-\frac{2 i t}{\sqrt{N}}+O\left(N^{-1}\right)}\left|\lambda_{1}\right\rangle+O\left(N^{-1 / 2}\right)\right] .
$$

At the time $T=\pi \sqrt{N} / 2$, we have $|\psi(T)\rangle=|w\rangle+$ $O\left(N^{-1 / 2}\right)$, i.e., the probability of success is one and the algorithm is optimal, as $T \propto \sqrt{N}$.

[19] R. Joynt, D. Zhou, and Q.-H. Wang, Int. J. Mod. Phys. B 25, 2115 (2011).

[20] M. A. C. Rossi and M. G. A. Paris, J. Chem. Phys. 144, 024113 (2016).

[21] C. Benedetti, F. Buscemi, P. Bordone, and M. G. A. Paris, Phys. Rev. A 87, 052328 (2013).

[22] E. Paladino, Y. M. Galperin, G. Falci, and B. Altshuler, Rev. Mod. Phys. 86, 361 (2014).

[23] C. Benedetti, F. Buscemi, P. Bordone, and M. G. A. Paris, Phys. Rev. A 93, 042313 (2016).

[24] I. Siloi, C. Benedetti, E. Piccinini, M. G. A. Paris, and P. Bordone, J. Phys.: Conf. Ser. 906, 012017 (2017).

[25] I. Siloi, C. Benedetti, E. Piccinini, J. Piilo, S. Maniscalco, M. G. A. Paris, and P. Bordone, Phys. Rev. A 95, 022106 (2017).

[26] E. Piccinini, C. Benedetti, I. Siloi, M. G. Paris, and P. Bordone, Comput. Phys. Commun. 215, 235 (2017).

[27] M. A. C. Rossi, C. Benedetti, M. Borrelli, S. Maniscalco, and M. G. A. Paris, Phys. Rev. A 96, 040301 (2017).

[28] Y. Yin, D. E. Katsanos, and S. N. Evangelou, Phys. Rev. A 77, 022302 (2008).

[29] A. Amir, Y. Lahini, and H. B. Perets, Phys. Rev. E 79, 050105 (2009).

[30] Z. Darázs and T. Kiss, J. Phys. A 46, 375305 (2013).

[31] E. Farhi and S. Gutmann, Phys. Rev. A 57, 2403 (1998).

[32] T. G. Wong, L. Tarrataca, and N. Nahimov, Quantum Inf. Proc. 15, 4029 (2016).

[33] J. Bezanson, A. Edelman, S. Karpinski, and V. B. Shah, SIAM Rev. 59, 65 (2017).

[34] M. Cattaneo and M. A. C. Rossi, QuantumSpatialSearch, https://github.com/matteoacrossi/QuantumSpatialSearch (unpublished).

[35] J. J. Sakurai, Modern Quantum Mechanics (Addison-Wesley, 1994). 